Grand Valley State University

ScholarWorks@GVSU

$12-2009$

\title{
A Pilot Study of an Automated Voice Response System and Nursing Intervention to Monitor Adherence to Oral Chemotherapy Agents
}

\author{
Veronica Decker \\ Cancer Care Associate \\ Sandra L. Spoelstra \\ Grand Valley State University, spoelsts@gvsu.edu \\ Emily Miezo \\ Michigan State University \\ Renee Bremer \\ Michigan State University \\ Mei You \\ Michigan State University
}

See next page for additional authors

Follow this and additional works at: https://scholarworks.gvsu.edu/kcon_articles

Part of the Medicine and Health Sciences Commons

\section{ScholarWorks Citation}

Decker, Veronica; Spoelstra, Sandra L.; Miezo, Emily; Bremer, Renee; You, Mei; Given, Charles W.; and Given, Barbara A., "A Pilot Study of an Automated Voice Response System and Nursing Intervention to Monitor Adherence to Oral Chemotherapy Agents" (2009). Peer Reviewed Articles. 29.

https://scholarworks.gvsu.edu/kcon_articles/29

This Article is brought to you for free and open access by the Kirkhof College of Nursing at ScholarWorks@GVSU. It has been accepted for inclusion in Peer Reviewed Articles by an authorized administrator of ScholarWorks@GVSU.

For more information, please contact scholarworks@gvsu.edu. 


\section{Authors}

Veronica Decker, Sandra L. Spoelstra, Emily Miezo, Renee Bremer, Mei You, Charles W. Given, and Barbara A. Given 
Title: A Pilot Study of an Automated Voice Response System and Nursing Intervention to Monitor Adherence to Oral Chemotherapy Agents

Short Title: Pilot Study on Adherence to Oral Chemotherapy

\author{
Authors: \\ Veronica Decker, MS, RN \\ Sandra Spoelstra, MSN, RN \\ Emily Miezo, MSN, RN \\ Renee Bremer, MS \\ Mei You, MS \\ Charles Given, PhD \\ Barbara Given, PhD, RN, FAAN
}

Email: veronica.decker@ht.msu.edu

Acknowledgements: This work was supported in part by the Walther Cancer Foundation, located in Indianapolis, Indiana.

\begin{abstract}
:
This study was designed to develop and test a system to monitor adherence with nonhormonal oral chemotherapeutic agents using an automated voice response (AVR) system plus nursing intervention. Participants received the Symptom Management Toolkit then participated in an interview for symptom severity, satisfaction, and beliefs about oral agents. Patients received weekly AVR calls, which assessed adherence to oral agents and severity of 15 symptoms. Patients who reported adherence of $<100 \%$ of
\end{abstract}


prescribed oral agents or symptoms of 4 or greater (0-10 scale) for three consecutive weeks, were called by a nurse for assistance with symptom management and adherence to oral chemotherapy medications. Following the 8 weekly AVR calls, patients participated in a follow up interview and medical record review. Subjects were 30 oncology patients who were ambulatory and treated at two cancer centers in Midwest USA. The results indicate $23.3 \%$ non adherence rate to oral chemotherapy medications due to symptoms and forgetting to take the medication. An association between symptom management and adherence was found. Symptom severity and beliefs about medications were not significantly different between adherent and non adherent patients. This pilot study demonstrated the ability to accrue patients for a longitudinal trial and informed intervention design, while providing guidance for future interventions and research studies.

\section{Background}

There are relatively few studies seeking to determine if patient adherence could be improved by reducing severity of symptoms and side effects thought to be related to certain medications. This pilot study was guided by two questions: 1) Can patients be monitored for adherence to oral chemotherapeutic agents and can adherence be improved by an Automated Voice Response (AVR) system and an intervention nurse where both address symptom management and adherence 2) Can this be demonstrated by an observed association between symptom management and improved patient adherence?

\section{Objectives}


The purpose of this study was to determine whether patients with breast, colon, or lung cancer, on non-hormonal oral chemotherapeutic agents can be monitored and receive interventions for adherence using an AVR system and a nurse intervener.

\section{Methods}

Setting/Sample: Accrual of patients for this pilot occurred at a National Cancer Institute Community Clinical Oncology Programs (NCI CCOP) clinical site, and a university based cancer clinic, both located in Michigan. Subjects were entered in the study between September 2007 and January 2008. Thirty subjects had solid tumor cancer diagnosis including breast, colon and lung cancers, and were on non-hormonal oral chemotherapeutic agents. Subjects had a touch-tone phone, no hearing deficits that interfered with using a telephone, understood English, had no cognitive deficits as evaluated by an on-site nurse, were willing to complete eight automated phone contacts requesting information on symptom severity and medication adherence, and phone contacts for nurse interventions for management of severity of symptoms and adherence to oral chemotherapeutic agents, and were not diagnosed with an emotional or psychological disorder under the care of a psychiatrist or psychologist. This pilot study began with trained nurse recruiters identifying subjects and explaining the study. All enrolled subjects received a copy of the Symptom Management Toolkit (SMT) from the nurse recruiter, prior to leaving the clinic.

\section{$\underline{\text { Instruments and Measures }}$}

Symptom Experience Inventory. The cancer symptom inventory, developed by this research team, has been used in previous work. ${ }^{2,3}$ Fifteen prevalent symptoms associated with patients undergoing chemotherapy were examined. Patients were asked if, within the past 7 days, they had experienced each symptom (yes/no) and, if so, to rate 
the severity on a 0 to10-point scale of how severe this symptom was and the extent the symptom disrupted or caused limitations in their regular daily activities.

Depression. The CESD-20 depression scale is a widely used measure of depressive affect for non-psychiatric populations. ${ }^{4,5}$ This measure has established psychometric properties (coefficient alpha .89 ). ${ }^{2}$

Short Form-12.The SF-12 functional ability scale is a widely used measure of an individual's ability to function. This measure has sound psychometric properties (alpha $.92)$ in this research.

Utilization and Services. The study focused on the use of increased physician oncology visits, hospitalizations, and emergency room visits during the study time period (8 weeks).

Beliefs About Medications; Satisfaction with Information on Oral Agents. Adherence to oral agents is related to patients' beliefs about the amount of information they have received regarding their prescribed oral chemotherapy agent, including actions and possible side effects, and with beliefs about concerns and necessity of specific medications. ${ }^{6,7}$ The concerns and necessities subscales to be used were developed from social cognitive ${ }^{8}$ and self-regulatory frameworks and represents potential costs associated with symptoms and side effects.

The Satisfaction with Information about Medicines Scale (SIMS) $)^{9}$ evaluates patients' perceptions of the quality of medication information that they have received. Two subscales related to medications were used. Internal consistency, measured by 
coefficient alpha for the oncology sample, was .81 and .80 respectively; test-retest reliability produced statistically significant correlations over time for each subscale; and validity as tested against adherence was significant.

The second set of measures, Beliefs about Medicines Questionnaire (BMQ), assesses patients' beliefs regarding the necessity and concerns about their medications. ${ }^{10}$ Specific items were tested across multiple samples of patients with different diseases. The specific "necessity" and "concerns" subscales had internal consistency scores in the mid 70's. Specific necessity correlated highly with adherence levels and concerns subscale correlated with poor adherence. This measure, while not tested on cancer patients, has strong psychometric properties and taps dimensions specific to the oral agents prescribed in this study.

Out-Of-Pocket Costs. At intake and 10 weeks, patients were asked how much they paid out-of-pocket (OOP) for their oral chemotherapy agents. These costs were then prorated over the numbers of pills purchased. The cost of the drugs was obtained from the pharmacies, and co-pays were determined for each oral agent.

Adherence with Oral Agents. Patient adherence to their oral chemotherapeutic agents was measured from multiple perspectives. Medical record audits were performed at the time the patient consented, (identify the site of cancer, stage, the name of the oral agent, the number of pills prescribed per day, times per day, number of pills per time, and the interval between doses) and at the end of the study (change in dose, pills per day intervals and dose delays, stoppages and the dates of each). Patient report of adherence was obtained from the intake and 10 week interviews and at each of the 8 AVR calls. During the intake and 10 week interviews, patients were asked to identify the 
name of their oral agent, number of pills to be taken in $\mathrm{AM}$ and $\mathrm{PM}$, and the number of days or weeks on and off pills per month or cycle. At each weekly AVR call, the system asked for the number of pills the patient took in the past seven days. If the patient reported a number different than programmed initially, the nurse intervener was sent an email notifying her of a discrepancy.

Specialty and Regular Pharmacy Report. Following the 10-week interview, the patient's pharmacy was contacted with the patient's permission and a report requested with fill and refill information for the oral chemotherapeutic agent(s), specifically detailing the number of pills dispensed to the patient during the study.

Calculation of Adherence Measures. The nurse intervener made the following comparisons: 1) compared information from intake audit with data collected during the intake interview on medications to assess the baseline level of adherence. This note was placed in the electronic intervention file for each patient and anchored decisions regarding adherence at subsequent calls. 2) Following each weekly call, patients reporting pill counts less or more than originally prescribed, received a call from the nurse intervener to confirm non-adherence and to provide an intervention when needed. If the patient reported a dose adjustment adjusted by the oncologist, then the nurse determined the reason for the adjustment and, if it was due to a symptom, assisted the patient to manage the symptom. At that point the intervention was logged as symptom assistance.

At the end of the 10-week study, the on-site nurse recruiter audited the medical chart, recorded dose changes, delays, reductions, stoppages, and toxic side effects and provided the audit results to MSU. This information was compared as follows: a) the 
weekly AVR patient reports of pill counts with the dose from the medical record adjusted for delays, interruptions, and stoppages; b) the Intake and 10-week interviews data were paired with the record data on dosing and changes as of the date of the interview; c) each of these indicators was paired at each time point against the availability of pills to the patient based upon the prescriptions filled and number of pills obtained per script. The pilot study collected an array of adherence measures for each patient.

Patient Acceptability and Satisfaction. Patient acceptability of the intervention was measured by number of AVR calls missed that were not related to hospitalization or poor health. Telephone hang-ups were counted and where in the course of the AVR script they occurred - symptom management or adherence. Second, acceptability was assessed by the proportion of nurse calls that were accepted and completed for management of symptoms and for assistance with adherence (nurse-related acceptance). Satisfaction with the AVR system and Symptom Management Toolkit (SMT) was measured using a short satisfaction instrument designed especially to capture patients' level of satisfaction with the AVR system that was used in the previous RCT. A series of satisfaction items describing dimensions of patients' satisfaction with the nurse calls were used to examine adherence. This satisfaction measure was used in a previous trial of the AVR. Psychometrics for this instrument were found to be acceptable (alpha greater than .65) as assessed during the course of the pilot study.

\section{Procedures}

The pilot study began with trained nurse recruiters identifying patients and explaining the study. All enrolled patients received a copy of the SMT from the nurse recruiter, prior to leaving the clinic. Enrolled patients then received an intake interview, eight AVR calls, an 
exit interview and nurse intervention calls when the AVR system indicated nonadherence and/or symptom severity of $\geq 4$ for three consecutive weeks.

\section{$\underline{\text { Interviews }}$}

Intake and Exit Interviews were conducted by experienced interviewers and entered onto a Computer Assisted Telephone Interview system. Sociodemographic information was collected on the intake interview. Patient intake and exit interviews included: measurements of adherence, compliance with oral medications, oral chemotherapy pill beliefs, health conditions, symptoms, the CESD, the SF-12, beliefs, and service utilization.

Data were collected at all eight AVR contacts by the investigation software. The AVR system used a pleasant female voice that asked to speak when that person would be available or the system called two more times at 40 minute intervals and repeated this process the following day beginning at the scheduled time. If no one answered on the second week, then the project manager contacted the patient directly.

The study patients received eight weekly calls from the AVR System to assess severity of 15 symptoms, and oral chemotherapy agent(s) adherence. At each contact, patients were asked to enter their personal identity number (PIN) for identification. If incorrect or forgotten, then someone from the staff called the patient and reminded them of their PIN so that calls could take place. At the first and all subsequent calls, patients were asked about the number of oral cancer pills they were prescribed and had actually taken in the past week. Then patients were queried as to the severity of each of 15 symptoms; asking them to indicate on a scale from 0 (not present) to 10 (worst severity possible), "currently how severe is your symptom?" At the end of the call the system indicated the 
symptoms that patients identified with a severity of 4 or higher and asked them to read those sections of the SMT for each symptom. Subsequent calls began with questions on the number of pills taken and then a review of those symptoms scored at a 4 or higher at the previous session then followed. Patients were asked: at the last call, you indicated (the voice states the symptom) symptom was a problem for you at a moderate or higher level; were you able to read the information in the SMT? If yes, how helpful was this information for managing this symptom?

Once all symptoms above threshold from the previous call were reviewed and the value of the SMT scored, the program asked the current adherence and symptom severity questions, severity of current symptoms rated, and the SMT assigned. To this script we added questions to patients regarding the numbers of pills they had taken in the past week and the number of pills prescribed. If the patient reported anything other than the exact number of chemotherapy pills prescribed, a nurse would review the pill count with the patient's prescription and a calendar indicating cycle information which included onoff days or weeks; and if the count appeared non-adherent the patient was called by the nurse to confirm an accurate pill count. Many times during this pilot patients either counted their pills incorrectly or pressed the wrong telephone keys. As a final means of confirming patient-adherence with the physicians prescribed dosage of oral chemotherapy, a medical record audit was conducted including pharmacy fill and refill information.

\section{Nursing Interventions}

Study patients reporting symptom severity at their weekly AVR calls were encouraged to use the SMT. In addition, if the patient reported anything less than $100 \%$ adherence through the weekly AVR calls or severity of one or more symptoms at $a \geq 4$ for three 
consecutive weeks, a nurse contacted the patient by telephone to confirm nonadherence and/or provided evidence-based nursing intervention for adherence and/or management of symptoms. Preliminary work using the AVR call system was completed by the research team comparing a nurse administered telephone intervention to reduce symptom severity among advanced cancer patients undergoing chemotherapy (primarily infusion). The results of the previous work indicated that both the AVR alone and with nursing intervention decreased symptom severity. ${ }^{11,12}$

Nursing interventions were based on Cognitive Behavioral Theory. The domains for the nursing interventions include: fostering self care behaviors, providing information about oral agents, problem solving for symptoms or adherence, providing support, coaching and counseling, communication with providers, and decision making; all to enhance tailored symptom management and adherence behaviors. The nurse intervention strategies were evidence-based for each symptom or adherence. Strategies on symptoms have been tested in three previous $\mathrm{NCl}$ funded RCTs and were used to form a plan of care for the patient. Symptom Management Toolkit (SMT) is a supplemental information source given to all patients. It is organized into a Frequently Asked Questions format and covers what is needed to manage side effects from treatment and the sequelae of cancer. Questions include: What is the symptom; How do people describe it; What causes it; What do people do to manage it; Where can they find additional information; and, Where can they find additional information? This guide has been used in several RCT's and is well accepted by patients. The reading level is $6^{\text {th }}-7^{\text {th }}$ grade.

\section{Results}


This pilot study examined the ability to monitor patients for adherence to oral chemotherapeutic agents, using an AVR program and a nurse administered telephone intervention to reduce symptom severity among advanced cancer patients undergoing chemotherapy. A total of 30 patients agreed to participate in the study, signed informed consents and completed the recruitment process. Eight withdrew from the study $(26.7 \%)$ for various reasons (predominately health related).

Sociodemographic Characteristics

The sociodemographics of the sample are presented in Table 1. The mean age of the subjects was 59.93 years $(S D=12.03)$, and $94 \%$ were female. Sixty-three percent of the subjects had at least some college education, $90 \%$ were white, and $80 \%$ were married. Breast cancer was the most common diagnosis and $77.67 \%$ were on a single oral chemotherapeutic agent, with varied lengths of time, from 1 to 8 months. No significant differences between the groups at baseline were found for gender, age, race, or ethnicity, education, marital status or type of cancer, and in some instances, the sample size was too small to test equivalency.

Non-adherence

The percent of missed pills was calculated weekly using the number of pills confirmed, divided by the number of pills prescribed. Seven out of 30 study subjects, or $23 \%$, had confirmed non-adherence over the eight week study period. The most common reported reason for non-adherence was 'forgot' to take the pills, which occurred with 5 subjects. One subject could not identify a reason of non-adherence. Six of the non-adherent patients missed pills one time out of the 8 weeks, or $13 \%$ of the time the subject missed their full number of prescribed pills. One patient missed 4 out of 8 weeks, or $50 \%$ of the time the subject missed their full number of prescribed pills. Of the six subjects who 
missed pills they took between $86 \%$ and $93 \%$ of their weekly pills. Of the subject that missed pills for 4 out of 8 weeks, the subject reported taking $90 \%$ of the prescribed pills on average.

Association between Symptom Management and Non-Adherence

To check the effect of symptom management, we used paired t-test to see how symptom severity changed before and after AVR intervention. Sum of symptom severity at intake was treated as symptom severity before intervention and at exit as after intervention. Overall, for 22 patients who finished intake, AVR intervention and the exit interview, their average sum of symptom severity decreased 4.35 with p-value 0.21 (which means not a significant difference between symptom severity before AVR intervention and after AVR intervention). When we compared the symptom severity before intervention and after intervention for those who stayed in the pilot study by their adherence status, there existed a marginal decrease in severity for non-adherence ( $p$ value $=0.04)$ as shown in Table 2

Association between Number of Oral Agents and Adherence To understand if two oral agents influence non-adherence more than one oral agent, a comparison was conducted (see Table 3 ). $77 \%$ of the study subjects were on one oral agent and $23 \%$ were on two oral agents. 17 study subjects, or $57 \%$, were on concurrent intravenous chemotherapy. $75 \%$ were adherent on single oral agent compared to $86 \%$ of the subjects who were on two oral agents. In addition, eight study subjects, or $27 \%$, discontinued their oral agents sometime during the study. Five study subjects or $17 \%$ had a physician initiated dosage change (increase or decrease in dosage).

Nurse Interventions 
During the pilot study, a specialty trained nurse intervener provided patient-centered personalized interventions via phone calls for patients that had symptom severity of 4 or higher for three consecutive weeks and/or to patients that had non-adherence of less than $100 \%$. (See Table 4)

Satisfaction Questionnaires

Satisfaction questionnaires were administered to all subjects following the 10 -week survey. $54 \%$ of the study subjects completed the satisfaction questionnaire, for a total of $77 \%$ (see Table 5). Of the 17 subjects that completed the satisfaction questionnaire, 12 subjects, or $70 \%$ were adherent, and 5 subjects, or $30 \%$ were non-adherent.

Overall, $100 \%$ of the subjects who completed the survey were either very satisfied or satisfied with the AVR for monitoring symptoms. Of those, $76 \%$ used the SMT with a high rate of referral to the appropriate section of the toolkit to manage a symptom. Of those who were contacted by a study nurse, $65 \%$ reported help from the nurse with symptoms and $100 \%$ reported help from the nurse with non-adherence. In summary, $60 \%$ felt the intervention was helpful, while $30 \%$ felt it was both burdensome and helpful, and $10 \%$ felt it was not helpful. Of those, $88 \%$ would recommend the intervention for symptom management and $53 \%$ for adherent to medications. Of the two subjects who were non-adherent and completed the survey, neither felt it was helpful with promoting adherence to the oral chemotherapy medication and they were non adherent for reasons of forgetting and missing pills.

\section{Discussion}

The primary aim of this study was to determine the feasibility of monitoring and providing interventions to patients with breast, colon, or lung cancer on non-hormonal 
chemotherapeutic agents sing and AVR system and nurse intervener. The results support such feasibility, as the system was easy to use, easy to learn, and captured the clinical information from patients in their homes.

A majority of patients experienced symptoms that were severe enough to generate referral to the SMT and nurse interventions, and non-adherence that prompted nurse interventions, as well as an alert to their clinician. Fatigue and pain were the most frequently occurring symptoms with symptom severity of $\geq 4$ for three consecutive weeks. Fatigue was a side effect of the oral agents that patients were on while on this study. Other less frequent symptoms warranting nurse intervention included lack of appetite, numbness and tingling, sleep disturbances and distress, which were also side effects from their oral agents (see Table 4). The secondary aims of this study were to determine if non-adherence can be defined and measured, to examine the relationship between symptom severity and adherence, and to compare and contrast the adherence group with the non-adherence group. The data presented here supports that contention.

First, and most importantly, non-adherence was defined and measured. Studies indicate that self-report measures, although the most common and easiest to use, have varied concordance with other means of measuring adherence. ${ }^{13}$ Self-reporting using the AVR system with follow-up from a nurse from the study would eliminate some of the inherent hesitancy a patient might have in admitting non-adherence to the clinicians that prescribed the medication. The patients who reported non-adherence through the AVR were confirmed when reported to the nurse. The research oncology nurse reviewed the enrollment documents, prior to each patient-call. The enrollment documents included oral agent information such as a calendar indicating when the patient was to be and off oral agent(s). The nurse asked each patient that reported non-adherence why they 
missed their pills and again as is consistent with other studies the most common reason for missed doses was forgetting. ${ }^{14}$ For the patient who reporting 'out of drug', we confirmed drug unavailability through a pharmacy audit. This appeared to be consistent with other studies, where the undersupply of cancer drugs appeared to be as common $(14 \%)$ as the undersupply of other drugs. ${ }^{15}$

Second, the AVR System was tested and found to work effectively and efficiently. Automated telecommunication systems have shown in published studies to improve medication adherence ${ }^{16}$ however to our knowledge this is the first time the AVR system has been used to explore how it might improve adherence to oral chemotherapeutic agents with cancer patients. We understand that one study showed that simple telephone contacts from the clinician's office by a non-nurse did not significantly improve adherence. ${ }^{17}$ However, the structured eight-week AVR system plus individualized nursing calls may more closely mirror the published work of Burke ${ }^{18}$ and Kim ${ }^{19}$ with the success of using behavioral interventions for improved adherence.

Finally, a beginning exploration of the relationship between symptom severity, adherence, and beliefs about medications occurred. Addressing the barriers to treatment adherence is complex and most likely patients have multiple reasons for non-adherence to oral agents. The severity of symptoms from cancer and the side effects of the oral agents may be one of the reasons. Studies of cancer and non-cancer diseases indicate that patients decrease adherence as symptoms and medication side effects occur. ${ }^{20}$ In this pilot study, no such effect was observed, most likely due to the small sample size.

Although it was beyond the scope of this pilot to evaluate the effectiveness of the AVR system and nurse calls as an intervention to improve adherence to oral 
chemotherapeutic agents, we did learn from our participants that our calls were perceived as 'caring.' One patient reported that no one at the clinic ever asked her if she was taking her pills and therefore she thought she was taking 'enough' although she missed four doses over the eight week period. Treatment adherence may be improved if clinicians directly ask patients about their adherence, as well as review the importance of taking oral medications as directed and the possible effect of adherence on their response to treatment. ${ }^{17-19,21,22}$

Future studies on interventions for non-adherence include asking the patient if they are taking their pills exactly as prescribed at each office visit. Perhaps this question will elicit a similar response to clinicians asking patients about their smoking cessation, where just asking the question at each visit will improve the outcome, in this case, improve adherence.

The level of satisfaction with and acceptability of the AVR was high. The technique, voice, and duration were acceptable to subjects and generally did not pose any technical difficulty. The few technical problems that were encountered were resolved in a timely fashion. Subjects offered suggestions on ways to expand and enhance the system that have already been incorporated into future versions.

With these revisions, the next research step, which has been initiated, is to test the value of the AVR symptom assessment and management system in improving adherence to oral chemotherapy agents. This study is ongoing and will further evaluate the relationship between symptom management and adherence. Before an electronic system can be incorporated into clinical care, it is essential to demonstrate that these systems are reliable, that they improve care, and that they are cost-effective. 


\section{Tables/Figures:}

\section{Table 1 Sociodemographic Characteristics and Cancer}

Adherence $(\mathrm{n}=\mathbf{2 3}$ or $\mathbf{7 7 \%}$ ) Non-adherence $(\mathrm{n}=\mathbf{7}$ or $23 \%)$

Demographic Characteristic

Gender

Male

Female

Age

21-40

$41-60$

$61-70$

$>71$

Race or ethnicity

White

Black

Asian/Other

\section{Education}

High school graduate

Some college

College graduate

Some post college

Marital status

Never married

Married

Divorced/Separated

Widowed

Type of Cancer

Breast Cancer
$2(8.7)$

21 (91.3)

7 (100.0)

1 (4.4)

11 (47.8)

5 (71.4)

4 (17.4)

2 (28.6)

7 (30.4)

$22(95.7))$

5 (71.4)

1 (4.4)

1 (14.3)

1 (14.3)

10 (43.5)

1 (14.4)

5 (21.7)

3 (42.3)

7 (30.4)

$3(42.3)$

1 (4.4)

-

2 (8.7)

19 (82.6)

5 (71.4)

2 (8.7)

2 (8.7)

$17(74.0)$

7 (100.0) 
Colon Cancer

$3(13.0)$

Lung Cancer

3 (13.0) 
Table 2. Summed Severity Scores on Intake and Exit Interviews

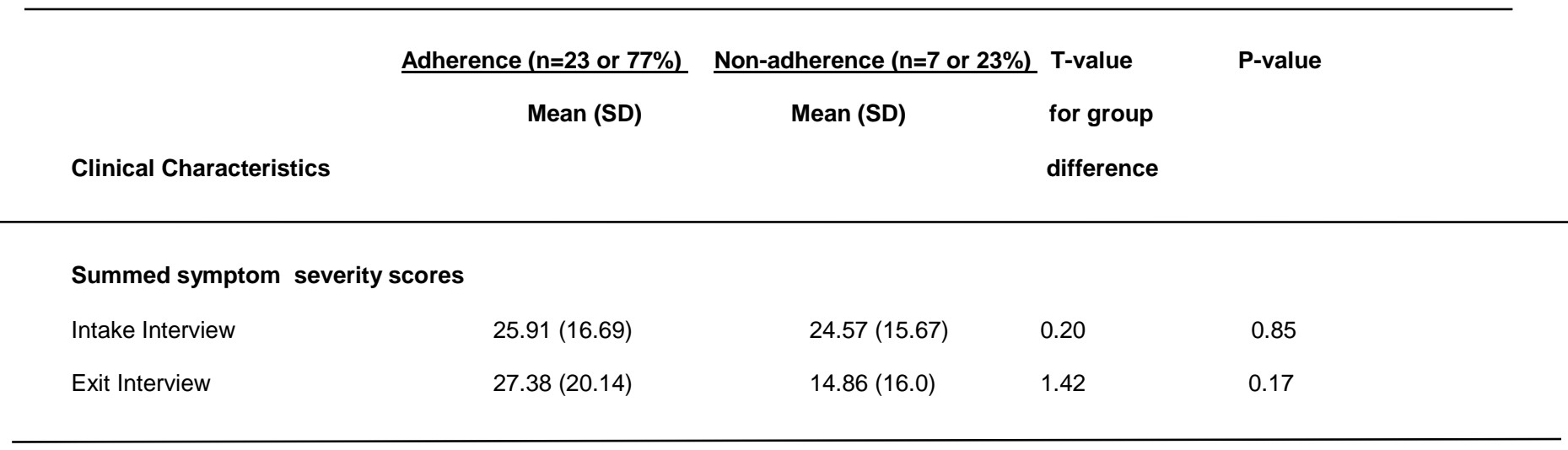

SD: standard deviation. 
Table 3. Oral Agents and Months on Oral Agent(s)

Adherence $(\mathrm{n}=\mathbf{2 3}$ or $\mathbf{7 7 \%})$

n $\%$

$12(70.6)$

$5(83.3)$

Capecitabine (Xeloda)

Cyclophosphamide (Cytoxan)

Erlotinib (Tarceva)

Lapatinib (Tykerb)

Type of multiple oral agent

Cyclophosphamide+Methotrexate

Capecitabine+Lapatinib

Months on oral agent

1 month
2 months
3 months
4 months
5 months
6 months
7 months
8 months

$2(11.8)$

$3(17.7)$

$-$

2 (33.3)

4 (66.7)

$8(38.1)$

5 (23.8)

$1(4.8)$

$1(4.8)$

$2(9.5)$

3 (14.3)

-

$1(4.8)$
$1(100.0)$

3 (42.9)

$1(14.3)$

1 (14.3)

2 (28.6)
Non-adherence ( $n=7$ or $23 \%$ )

n $\%$ 
Table 4. Number of Patients with Symptoms above Threshold (>=4), AVR Interventions,

Nurse Interventions, Resolution of Symptom by Adherent and Non-adherent Patients

\begin{tabular}{|c|c|c|c|c|c|}
\hline & Number of & Number of & Number of & Number of & Total Number \\
\hline & Patients & Patients & Patients & Patients & of Patients \\
\hline & Severity $>=4$ at & Severity Resolved & Nurse & Nurse with & Symptoms by \\
\hline & First Contact & by $3^{\text {rd }}$ week & $\mathrm{N}$ & Resolved Symptom & End of Study \\
\hline \multicolumn{4}{|l|}{ Symptoms } & \multicolumn{2}{|l|}{$N(\% * *)$} \\
\hline Fatigue & $12(40.00)$ & $4(33.33)$ & 8 & 2 of $8(25.00)$ & 6 of $11(50.00))$ \\
\hline Nausea/vomiting & $3(10.00)$ & $1(33.33)$ & 2 & 1 of $2(50.00)$ & 2 of $3(66.67)$ \\
\hline Lack of appetite & $8(26.67)$ & $4(50.00)$ & 4 & 2 of $4(50.00)$ & 6 of $8(75.00)$ \\
\hline Constipation & $5(16.67)$ & $2(40.00)$ & 3 & 1 of $3(33.33)$ & 3 of $5(60.00)$ \\
\hline Skin rash & $6(20.00)$ & $5(83.33)$ & 1 & 1 of $1(100)$ & 6 of $6(100)$ \\
\hline Numbness/tingling & $9(30.00)$ & $5(55.56)$ & 4 & 1 of $4(25.00)$ & 6 of $9(66.67)$ \\
\hline Sleep disturbance & $4(13.33)$ & 0 & 4 & 2 of $4(50.00)$ & 2 of $4(50.00)$ \\
\hline Sore mouth & $5(16.67)$ & $4(80.00)$ & 1 & 0 & 4 of $5(80.00)$ \\
\hline
\end{tabular}

*Percentage based on number of patients with symptoms above threshold at first contact

** Percentage based on number of patients referred to nurse intervention 
Table 5. Satisfaction with the AVR, SMT, and Nurse Administered Interventions

Adherence $(n=1270.58 \%)$ Non-adherence $(n=529.42 \%)$

Satisfaction Questions $\quad n\left(\% \%^{* *}\right) \quad n\left(\%{ }^{* *}\right)$

\section{AVR for monitoring symptoms}

Very satisfied

Somewhat satisfied

Just right

Use of SMT

Yes

No

Telephone referred to appropriate

Section of SMT to manage symptom *

Strongly agree

Agree

Neither agree or disagree

Disagree

Telephone helped patient be more independent for symptom management *

Strongly agree

Agree

Neither agree or disagree

Disagree

The MSU nurse helped with symptoms *

Strongly agree

Agree

Neither agree or disagree
$9(75.00)$
$1(20.00)$
$2(40.00)$
$3(25.00)$
2 (40.00)

$9(75.00)$

$4(80.00)$

$3(25.00)$

$1(20.00)$
$2(16.67) \quad 1(25.00)$

4 (33.33) $\quad 3(75.00)$

$3(25.00)$

$3(25.00)$ 
Disagree

$2(20.00)$

$1(25.00)$

The MSU nurse helped with adherence *

Strongly agree

Agree

Neither agree or disagree

Disagree

Strongly disagree

Overall AVR and Oral Chemotherapy

Monitoring system

Burdensome

Both burdensome and helpful

Helpful

Recommend AVR to Oncologist to

Monitor symptoms *

Yes

No

\section{Recommend AVR to Oncologist to}

\section{Remind to take pills}

Yes

7 (58.33)

$5(41.67)$

No
$2(40.00)$

5 (41.67)

7 (58.33)

$3(60.00)$
$10(91.91)$
$4(80.00)$
$1(9.09)$
$1(20.00)$

* Some respondents did not complete the entire survey.

** Percentage totaled for each adherence and non-adherence group. 
Key Words: Oral Chemotherapy. Symptom management. Adherence.

\section{References}

${ }^{1}$ DiMatteo MR, Giordani PJ, Lepper HS, et al: Patient adherence and medical treatment outcomes: A Meta-analysis. Medical Care 40:794-811, 2002

${ }^{2}$ Giesler RB, Given B, Given CW, et al: Improving the quality of life of patients with prostate carcinoma: a randomized trial testing the efficacy of a nurse-driven intervention. Cancer 104:752-62, 2005

${ }^{3}$ Given B, Given CW, McCorkle R, et al: Pain and fatigue management: Results of a nursing randomized clinical trial. Oncology Nursing Forum 29:949-56, 2002

${ }^{4}$ Radloff L: The CES-D Scale: A Self-Report Depression Scale for Research in the General Population. Applied Psychological Measurement 1(3): 385-401, 1977

${ }^{5}$ Rawl S, Given B, Given CW, et al: Effects of advanced practice nursing interventions on physical and psychological functioning for newly diagnosed cancer patients. Oncology Nursing Forum, In Press

${ }^{6}$ Horne R, Weinman J: Patients' Beliefs about Prescribed Medicine and Their Role in Adherence to Treatment in Chronic Physical Illness. Journal of Psychosomatic Research 47(6): 555-567, 1999

${ }^{7}$ Horne, R: Patients' Beliefs about Treatment: The Hidden Detriment of Treatment Outcome? 47(6): 491-495, 1999

${ }^{8}$ Azjen I: From Intentions to Actions: A Theory of Planned Behaviour. Heidelberg, Springer-Verlag, 1985

${ }^{9}$ Horne R, Hankins M, Jenkins R: The Satisfaction with Information about Medicines Scale (SIMS): a new measurement tool for audit and research. Qual Health Care 10:135-40, 2001 
${ }^{10}$ Horne R, Weinman J, Hankins M: The beliefs about medicines questionnaire: The development and evaluation of a new method for assessing the cognitive representation of medication. Psychology and Health 14:1-24, 1999

${ }^{11}$ Kurtz ME, Kurtz JC, Given CW, et al: Effects of a symptom control intervention on utilization of health care services among cancer patients. Medical Science Monitor 12:CR319-24, 2006

${ }^{12}$ Sikorskii A, Given CW, Given B, Jeon S, Decker V, Decker D, Champion V, McCorkle R. Symptom management for cancer patients: a trial comparing two multimodal interventions. Journal of Pain Symptom Management 34(3):253-64, 2007

${ }^{13}$ Garber M, Nau DP, Erickson S, et al: The concordance of self-report with other measures of medication adherence: A summary of the literature. Medical Care 42:649-652, 2004

${ }^{14}$ Conn VS, Taylor SG, Stineman A: Medication management by recently hospitalized older adults. Journal of Community Health Nursing 9:1-11, 1992

${ }^{15}$ Nilsson KL, Andersson K, Bergkvist A, et al: Refill adherence to repeat prescriptions of cancer drugs to ambulatory patients. Journal of Cancer Care 15:235-237, 2006

${ }^{16}$ Friedman $\mathrm{RH}$, Kazis LE, Jette AM, et al: A telecommunications system for monitoring and counseling patients with hypertension: Impact on medication adherence and blood pressure control. American Journal of Hypertension 9:285-292, 1996

${ }^{17}$ Schectman G, Hiatt J, Hartz A: Telephone contacts do not improve adherence to niacin or bile acid sequestrant therapy. The Annals of Pharmacotherapy 28:2934, 1994

${ }^{18}$ Burke LE, Dunbar-Jacob J, Orchard TJ, et al: Improving adherence to a cholesterollowering diet: A behavioral intervention study. Patient Education \& Counseling $57: 134-142,2005$ 
${ }^{19} \mathrm{Kim}$ HS, Oh JA: Adherence to diabetes control recommendations: Impact of nurse telephone calls. Journal Advanced Nursing 44:256-261, 2003

${ }^{20}$ Schlenk EA, Dunbar-Jacob J, Engberg S: Medication non-adherence among older adults: A reveiw of strategies and interventions for improvement. Journal of Gerontological Nursing July: 33-43, 2004

${ }^{21}$ Haynes RB, McDonald H, Garg A: Helping Patients Follow Prescribed Treatment: Clinical Applications. JAMA 222(22): 2880-2883, 2002

${ }^{22}$ Kripalani S, Yao X, Haynes RB: Interventions to Enhance Medication Adherence in Chronic Medical Conditions: A Systematic Review. Arch Intern Med 167: 540550,2007 